\title{
Regiodivergent Biocatalytic Hydroxylation of L-Glutamine Facilitated by Characterization of Non-Heme Dioxygenases from Non-Ribosomal Peptide Biosyntheses
}

\author{
Hans Renata*, Emily Shimizu, Christian R. Zwick, III
}

The Scripps Research Institute, Department of Chemistry, 130 Scripps Way, Jupiter, FL 33458,

USA

hrenata@scripps.edu

\begin{abstract}
We report the functional characterization of two iron- and $\alpha$-ketoglutarate-dependent dioxygenases that are capable of hydroxylating free-standing glutamine at its $\mathrm{C} 3$ and $\mathrm{C} 4$ position respectively. In particular, the $\mathrm{C} 4$ hydroxylase, $\mathrm{Q} 4 \mathrm{Ox}$, catalyzes the reaction with approximately 4,300 total turnover numbers, facilitating synthesis of a solid-phase compatible building block and stereochemical elucidation at the $\mathrm{C} 4$ position of the hydroxylated product. This work will enable the development of novel synthetic strategies to prepare useful glutamine derivatives and stimulate further discoveries of new amino acid hydroxylases with distinct substrate specificities.
\end{abstract}

\section{Introduction}

Amino acids are some of the most indispensable chemicals in biological systems and modern drug discovery efforts. ${ }^{1}$ In addition to their incorporation into peptides and other biological macromolecules, amino acids also serve as precursors to amine-based primary metabolites and neurotransmitters. Their unique three-dimensionality, combined with the presence of useful and diverse functional groups, render amino acids highly desirable building blocks in combinatorial synthesis. Recent renaissance in peptide drug discovery ${ }^{2}$ has spurred the development of new methods to access novel amino acid structures that go beyond the twenty proteinogenic 
counterparts. Among the various possible modifications, $\mathrm{C}-\mathrm{H}$ hydroxylation of proteinogenic amino acids (pAAs) can play an important role in modulating the biological and pharmacological properties of the resulting structures. For example, C3 hydroxylation of prolines in Gly-Pro-Pro repeats in collagen ${ }^{3}$ play a key role in regulating its tertiary structure and stability. Additionally, 4-hydroxy-L-isoleucine has been described to display antidiabetic and antiobesitic activities. ${ }^{4}$ Hydroxylation of pAAs can also act as a gateway for further modifications, ${ }^{5}$ as exemplified by the biogenesis of 4-methyl-L-proline from L-leucine ${ }^{6}$ and the recent discovery that the biosynthesis of ibotenic acid begins by selective C3-hydroxylation of glutamate. ${ }^{7}$ Synthetic chemistry similarly views the alcohol moiety on hydroxylated amino acids as a useful chemical handle for further structural diversification. Some of the methods commonly employed for such diversification include fluorine incorporation via nucleophilic substitution ${ }^{8}$ and $\mathrm{C}-\mathrm{C}$ bond formation via oxidation/methylenation sequence. ${ }^{9}$

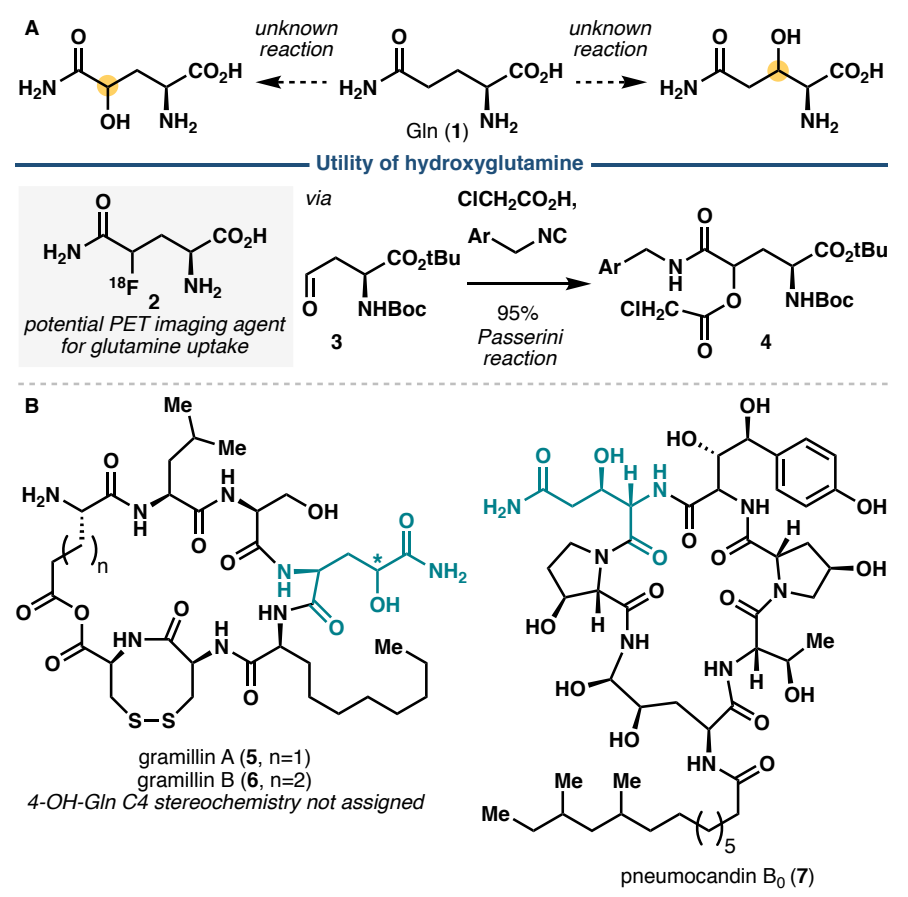


Figure 1. A. Utility of glutamine derivatives (e.g., 2) and the current gap in knowledge for direct functionalization of glutamine (1). B. Representative examples of nonribosomal peptide natural products with hydroxylated glutamine motif.

Given the importance of hydroxylated amino acids, our laboratory has developed a research program aimed at identifying useful oxygenases for regio- and stereoselective $\mathrm{C}-\mathrm{H}$ hydroxylation of readily available amino acids. To date, our efforts have led to the discovery of a leucine 5hydroxylase, GriE, ${ }^{10}$ capable of effecting selective $\mathrm{C} 5$ hydroxylation on a range of aliphatic amino acids and two additional hydroxylases, $\mathrm{GlbB}^{11}$ and GetI, ${ }^{12}$ that natively catalyze selective $\mathrm{C} 4$ hydroxylation on lysine and citrulline, respectively. Despite this success and efforts from other laboratories, no dedicated that catalyzes selective $\mathrm{C}-\mathrm{H}$ hydroxylation on free-standing glutamine (Gln) has been identified to date. The hydroxylated product from this transformation is a useful chemical, especially for the production of fluorine-18 labeled glutamine derivatives as positron emission tomography (PET) imaging agents. Seminal work from Qu and Kung in $2010^{13}$ showed the viability of using derivative 2 to image glutamine uptake in SF188Bcl-xL tumor cells, which are glutamine addicted derivative of glioblastoma cells. Prior synthesis of $\mathbf{2}$ commenced from partially-reduced aspartate derivative $\mathbf{3}$, which was subjected to a three-component Passerini reaction to install the $\mathrm{C} 5$-amide of the target molecule. An alternative approach to $\mathrm{C} 4$-hydroxylated Gln was reported by Brimble and co-workers in 2017, involving the use of C4-hydroxylated proline derivative, which was oxidized to the corresponding C5 amide and then opened to the linear form via ammonolysis. ${ }^{14}$ To identify a more direct access to hydroxylated glutamine derivatives, we sought to identify complementary biocatalytic methods for regioselective $\mathrm{C}-\mathrm{H}$ oxidations of free-standing glutamine. 


\section{Results and Discussion}

To identify potential enzyme candidates, we noted the presence of hydroxylated glutamine motifs in several nonribosomal peptides, namely the gramillins $(\mathbf{5}, \mathbf{6}),{ }^{15}$ pneumocandin $\mathrm{B}_{0}(\mathbf{7})^{16}$ and fellutamide $A \cdot{ }^{17}$ Among the biosynthetic gene clusters (BGCs) for these natural products, we observed that those corresponding to the gramillins and pneumocandin $\mathrm{B}_{0}$ contain genes encoding for iron- and $\alpha$-ketoglutarate-dependent dioxygenases ( $\mathrm{Fe} / \alpha \mathrm{KGs}$ ). We first sought to characterize the gene product of FGSG_00048 (Uniprot ID: I1R9B5), the gene encoding for Fe/ $\alpha \mathrm{KG}$ from the gramillin $\mathrm{BGC}$ due to its potential implication in the production of 4-OH-Gln, which is more valuable synthetically than 3-OH-Gln, especially in light of recent discoveries of $\mathbf{2}$ as a viable PET imaging agent and $\mathrm{IboH}$ as an $\mathrm{Fe} / \alpha \mathrm{KG}$ glutamate $\mathrm{C} 3$ hydroxylase. At the outset, the role of the gene product of FGSG_00048 is unclear though Bahadoor and Harris had hypothesized that it might be involved in the biosynthesis of the 2-amino decanoic acid monomer and that a P450 might be responsible for the production of 4-OH-Gln. Furthermore, BLAST analysis of FGSG_00048 did not reveal any significant sequence similarity to any known amino acid hydroxylases. Finally, even if the gene product is indeed involved in C4 hydroxylation of the Gln monomer, there are still two biosynthetic scenarios to be considered, namely (a) hydroxylation occurs on the free-standing monomer prior to its loading to the NRPS module, or (b) hydroxylation takes place after the cyclic peptide is fully assembled by the NRPS module.

To facilitate functional characterization, $F G S G \_00048$ was codon optimized and inserted into pET28 expression vector. Heterologous expression of its gene product, hereby named Q4Ox, produced soluble enzyme, which upon in vitro assay in the presence of $\mathrm{Fe}^{2+}, \alpha \mathrm{KG}$ and $\mathrm{O}_{2}$, validated its function as glutamine $\mathrm{C} 4$ hydroxylase. No hydroxylated product could be observed 
when $\mathrm{Fe}^{2+}$ or $\alpha \mathrm{KG}$ was omitted from the reaction, which confirmed that $\mathrm{Q} 4 \mathrm{Ox}$ belongs to the ironand $\alpha$-ketoglutarate-dependent dioxygenase superfamily. Further analysis showed that the hydroxylation reaction proceeded in a highly efficient fashion (4,300 total turnover number) and with complete diastereoselectivity. This observation was validated with steady-state kinetic analysis, which afforded a $k_{\text {cat }}$ of $250 \pm 2.6 \mathrm{~min}^{-1}$ and a $K_{\mathrm{M}}$ of $0.99 \pm 0.03 \mathrm{mM}$. These parameters are within the range of those obtained from other amino acid hydroxylases characterized in our lab in recent years. Testing of other pAAs in the reaction revealed a highly narrow substrate specificity as no other amino acids were accepted as substrate in the reaction. This discovery also suggests that hydroxylation of Gln in gramillin biosynthesis occurs early in the pathway, prior to its loading to the NRPS module and macrocyclization.

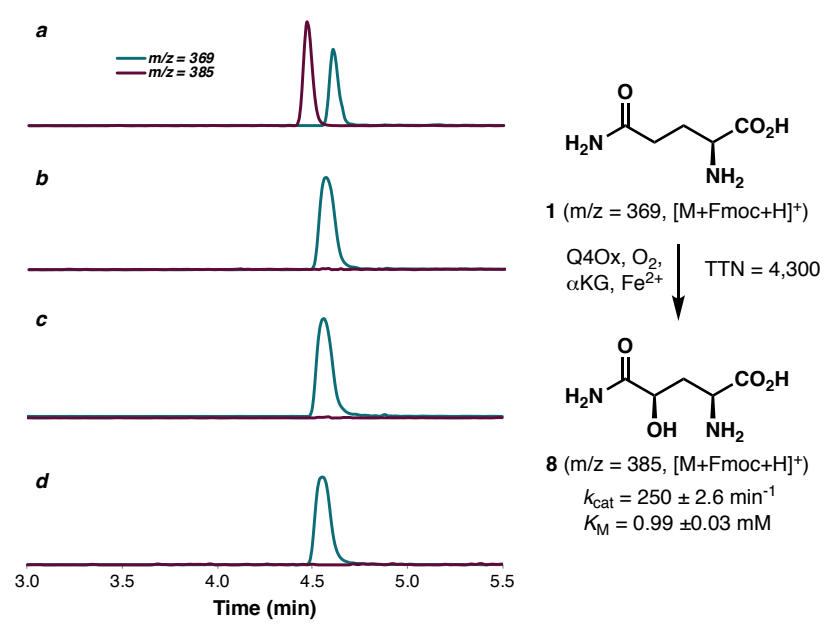

No reaction with other amino acids: Glu, Asn, Asp, Arg, Cit, Lys, Orn, Leu, Ile, Val

Figure 2. LCMS profile of glutamine hydroxylation with Q4Ox following Fmoc derivatization; (a) in the presence of $\mathrm{FeSO}_{4}, \alpha \mathrm{KG}$, ascorbic acid and enzyme; (b) in the absence of enzyme; (c) in the absence of $\mathrm{FeSO}_{4}$; (d) in the absence of $\alpha \mathrm{KG}$.

We next sought to demonstrate the practicality of this reaction in a preparative scale biotransformation. On $100 \mathrm{mg}$ scale, $\mathrm{C}-\mathrm{H}$ hydroxylation of $\mathrm{Gln}$ in the presence of purified Q4Ox 
proceeded with complete conversion and the desired product could be isolated cleanly through ion-exchange purification. The same reaction could also be performed with lysates of E. coli expressing Q4Ox with similar levels of efficiency, thereby bypassing the need for tedious protein purification. It is worth noting that reaction with lysates proved sensitive to reaction scale, lysis conditions and headspace volume and that minor amounts of transamination products between Gln and $\alpha \mathrm{KG}$, likely catalyzed by endogenous transaminases in $E$. coli lysates, could be observed when small deviations to these parameters were introduced. Initial efforts to derivatize $\mathbf{8}$ were hampered by solubility issues. Furthermore, the ion-exchange purification procedure consistently led to only $80-85 \%$ mass recovery. To circumvent these issues, we developed a telescoped protocol to generate Fmoc-protected 4-OH-Gln (9) which bypassed the isolation of the free amino acid product. Upon completion of the enzymatic hydroxylation step, a solution of 9-fluorenylmethylsuccinimidyl carbonate (Fmoc-OSu) in acetonitrile could simply be added to effect Fmoc protection under biphasic conditions, providing 9 in moderate yield on preparative scale $(100 \mathrm{mg})$. In our previous work, we found that several C4- and C5-hydroxylated amino acids displayed high propensity towards self-lactonization ${ }^{10,11}$ during protection of their $\alpha$-amino group. In contrast, 9 did not possess such tendencies and was observed to remain as the open-chain form in organic solvent or upon storage at ambient temperature. This unique feature of $\mathbf{9}$ was leveraged in the synthesis of a solid-phase peptide synthesis-compatible building block 10 via TBS protection of the secondary alcohol. To elucidate the stereochemical configuration at $\mathrm{C} 4$ of the hydroxylated Gln product, we converted $\mathbf{8}$ to protected derivative $\mathbf{1 1}$ and compared its ${ }^{1} \mathrm{H}$ and ${ }^{13} \mathrm{C}$ NMR spectra to a related compound 11', which was used by Brimble and co-workers as a key intermediate towards hemerocallisamine $\mathrm{I} .{ }^{14}$ Several key differences in chemical shifts and ${ }^{1} \mathrm{H}$ splitting patterns were noted between the two structures, suggesting that they exist in a diastereomeric relationship. 
Based on this observation, we propose that $\mathbf{1 1}$ exists in a $(2 S, 4 R)$-configuration and as a corollary, the modified Gln motif in the gramillins likely exists in a $(2 S, 4 R)$-configuration as well.

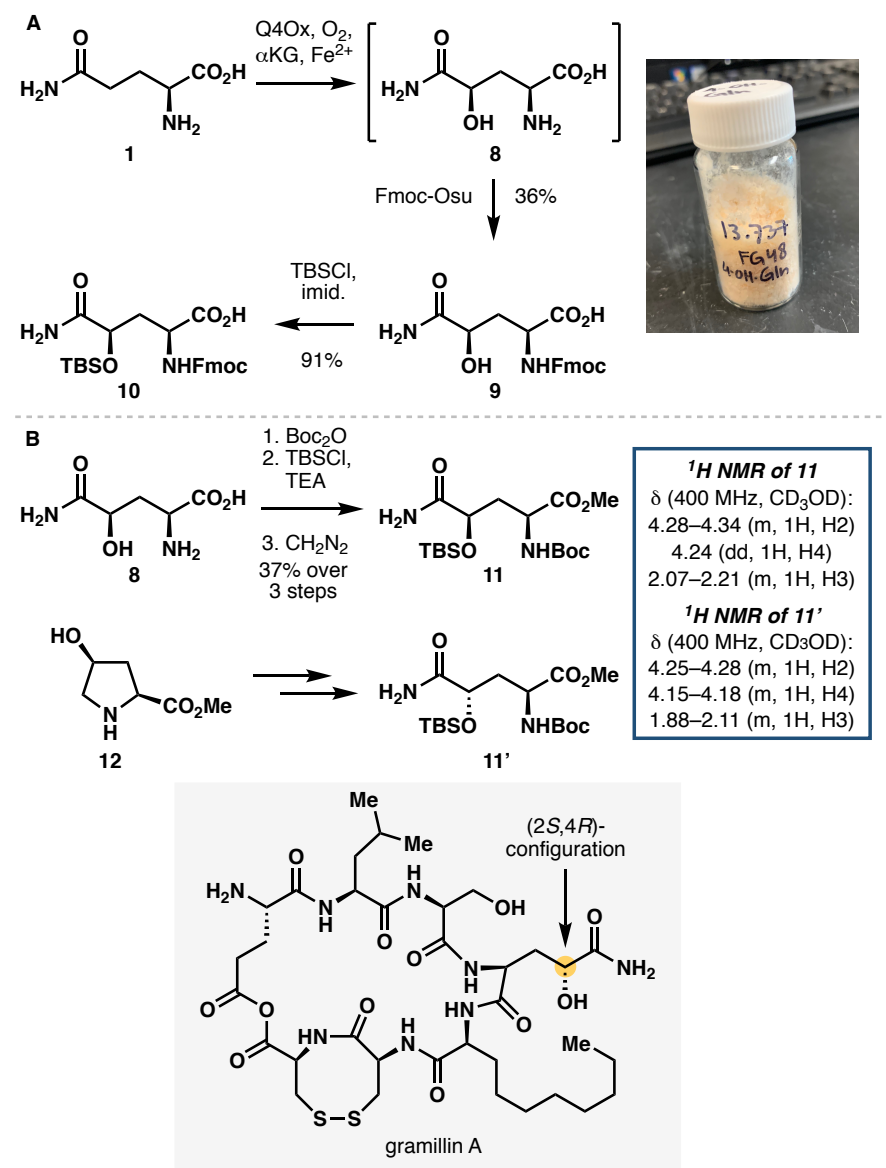

Figure 3. A. Derivatization of $4-\mathrm{OH}-\mathrm{Gln}(\mathbf{8})$ to a solid-phase peptide synthesis-compatible building block 10. B. Determination of stereochemical configuration of $\mathbf{8}$ via derivatization and ${ }^{1} \mathrm{H}$ NMR comparison with known compound 11'.

Following the above work, we turned our focus to the functional characterization of GloE (Uniprot ID: S3E7Q2). Our effort in this area was greatly enabled by pioneering report from Tang and Walsh, who had previously characterized several Fe/ $\alpha$ KGs from echinocandin B biosynthesis. ${ }^{18}$ While the $\mathrm{BGC}$ of pneumocandin $\mathrm{B}_{0}$ also contains multiple $\mathrm{Fe} / \alpha \mathrm{KG}$-encoding genes, no homologs of GloE could be found in the BGC of echinocandin B, which differs from the pneumocandins by 
the substitution of the 3-OH-Gln unit with Thr. Based on this observation, we hypothesized that GloE is responsible for the production of the 3-OH-Gln monomer in pneumocandin $\mathrm{B}_{0}$. At this stage, however, it was unclear whether GloE would act on a free-standing amino acid or one that is bound to a peptidyl carrier protein. GloE could be solubly expressed in E. coli as an N-His $6^{-}$ tagged protein. In vitro assay of GloE in the presence of $\mathrm{Gln}, \alpha \mathrm{KG}, \mathrm{Fe}^{2+}$ and molecular oxygen, followed by Fmoc derivatization revealed the presence of a new species with an $\mathrm{m} / \mathrm{z}$ corresponding to hydroxylated Gln. Though only modest total turnover number $(\mathrm{TTN}=44)$ could be observed, the reaction could be scaled up sufficiently to support ${ }^{1} \mathrm{H}$ NMR analysis, which provides conclusive evidence for hydroxylation of Gln at C3. To probe the possibility that GloE might act on peptidyl-carrier-bound amino acid substrate, we also attempted in vitro assay with $\mathrm{N}$ acetylcysteamine derivative of $\mathrm{Gln}(\mathrm{Gln}-\mathrm{SNAc}, \mathbf{1 4})$ as a substrate mimic ${ }^{19}$ of carrier-protein-bound Gln. However, no reaction could be observed with this substrate, suggesting that free Gln is the likely native substrate of GloE. Unfortunately, due to the low activity of GloE, accurate determination of its steady-state kinetic parameters was not possible. Though the low activity of GloE might seem surprising for a secondary metabolite biosynthetic enzyme, it is worth noting that several Fe/ $\alpha$ KGs from echinocandin B biosynthesis also exhibited low TTNs on their native substrates before undergoing self-inactivation. ${ }^{18}$ Finally, reaction with lysates of E. coli expressing GloE was attempted, but due to the modest activity of GloE, the competing transamination pathway, previously described in the reaction with Q4Ox, predominated. 


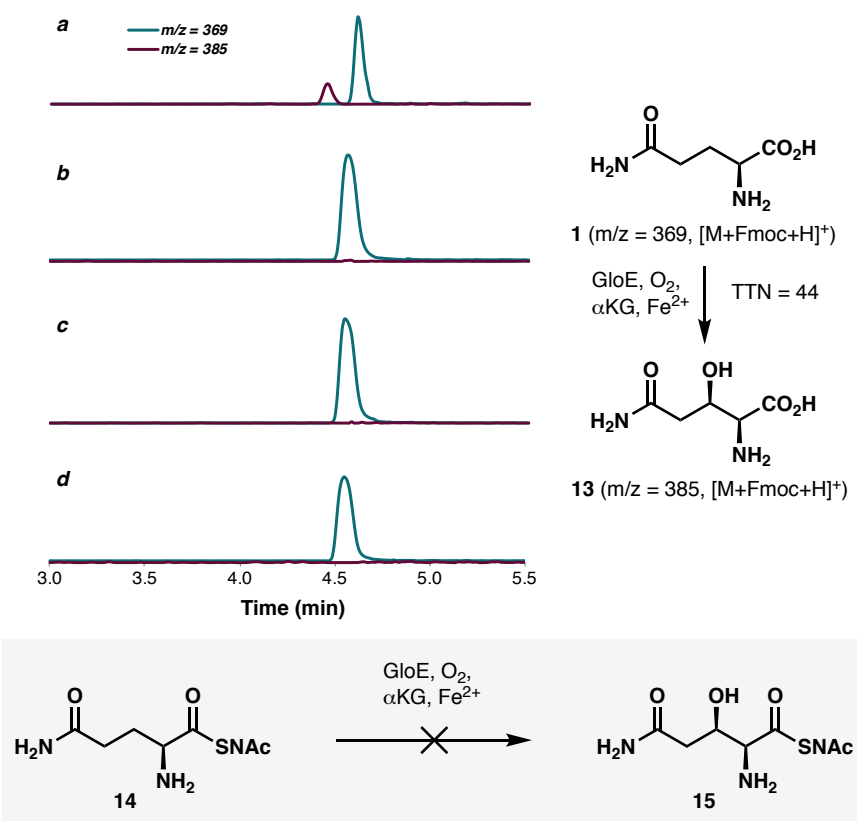

Figure 4. LCMS profile of glutamine hydroxylation with GloE following Fmoc derivatization; (a) in the presence of $\mathrm{FeSO}_{4}, \alpha \mathrm{KG}$, ascorbic acid and enzyme; (b) in the absence of enzyme; (c) in the absence of $\mathrm{FeSO}_{4}$; (d) in the absence of $\alpha \mathrm{KG}$.

\section{Conclusion}

In this work, we described the functional characterization of two new glutamine hydroxylases, Q4Ox and GloE, belonging to the gramillins and pneumocandin $\mathrm{B}_{0}$ biosynthetic pathways, respectively. Both enzymes exhibited strict substrate specificity towards Gln and while the hydroxylation activity of GloE is only modest, Q4Ox is able to catalyze the conversion of Gln to 4-OH-Gln in a highly efficient fashion. Biocatalytic hydroxylation with Q4Ox could be performed on preparative scale, allowing sufficient material throughput for further product derivatization to afford a building block that is anticipated to be compatible with solid-phase peptide synthesis. This work further expands the biocatalytic toolbox for selective $\mathrm{C}-\mathrm{H}$ functionalization of amino acids 
and paves the way for the development of a biocatalytic-centered synthetic strategy to access ${ }^{18} \mathrm{~F}$ derivative of Gln for PET tracer studies.

\section{Declaration of Competing Interest}

No conflict of interest.

\section{Acknowledgments}

Financial support for this work was provided by the National Institutes of Health (Grant R35 GM128895). We thank Prof. Margaret Brimble for sharing the NMR spectra of compound 11'. We acknowledge the Kodadek and Shen labs for generous access to their instrumentations.

\section{References}

1. Blaskovich, M. A. T. Unusual Amino Acids in Medicinal Chemistry. J. Med. Chem. 2016, 59, 10807-10836.

2. Henninot, A.; Collins, J. C.; Nuss, J. M. The Current State of Peptide Drug Discovery: Back to the Future? J. Med. Chem. 2018, 61, 1382-1414.

3. Jenkins, C. L.; Bretscher, L. E.; Guzei, I. A.; Raines, R. T. Effect of 3-Hydroxyproline Residues on Collagen Stability. J. Am. Chem. Soc. 2003, 125, 6422-6427.

4. Sauvaire, Y.; Petit, P.; Broca, C.; Manteghetti, M.; Baissac, Y.; Fernandez-Alvarez, J.; Gross, R.; Roye, M.; Leconte, A.; Gomis, R.; Ribes, G. 4-Hydroxyisoleucine: A Novel Amino Acid Potentiator of Insulin Secretion. Diabetes 1998, 47, 206-210.

5. Hedges, J. B.; Ryan, K. S. Biosynthetic Pathways to Nonproteinogenic $\alpha$-Amino Acids. Chem. Rev. 2020, 120, 3161-3209. 
6. Luesch, H.; Hoffmann, D.; Hevel, J. M.; Becker, J. E.; Golakoti, T.; Moore, R. E. Biosynthesis of 4-Methylproline in Cyanobacteria: Cloning of $\operatorname{nos} E$ and $\operatorname{nos} F$ Genes and Biochemical Characterization of the Encoded Dehydrogenase and Reductase Activities. $J$. Org. Chem. 2003, 68, 83-91.

7. Obermaier, S.; Müller, M. Ibotenic Acid Biosynthesis in the Fly Agaric Is Initiated by Glutamate Hydroxylation. Angew. Chem. Int. Ed. 2020, 59, 12432-12435.

8. Rentmeister, A.; Arnold, F. H.; Fasan, R. Chemo-Enzymatic Fluorination of Unactivated Organic Compounds. Nat. Chem. Biol. 2009, 5, 26-28.

9. Murphy, A. C.; Mitova, M. I.; Blunt, J. W.; Munro, M. H. G. Concise, Stereoselective Route to the Four Diastereomers of 4-Methylproline. J. Nat. Prod. 2008, 71, 806-809.

10. Zwick, C. R., III; Renata, H. Remote C-H Hydroxylation by an a-Ketoglutarate-Dependent Dioxygenase Enables Efficient Chemoenzymatic Synthesis of Manzacidin C and Proline Analogs. J. Am. Chem. Soc. 2018, 140, 1165-1169.

11. Amatuni, A.; Renata, H. Identification of a Lysine 4-Hydroxylase from the Glidobactin Biosynthesis and Evaluation of Its Biocatalytic Potential. Org. Biomol. Chem. 2019, 17, $1736-1739$.

12. Zwick, C. R., III; Sosa, M. B.; Renata, H. Characterization of a Citrulline 4-Hydroxylase from GE81112 Biosynthesis and Engineering of Its Substrate Specificity for the Chemoenzymatic Synthesis of Enduracididine. Angew. Chem. Int. Ed. 2019, 58, 1885418858.

13. Qu, W.; Zha, Z.; Ploessl, K.; Lieberman, B. P.; Zhu, L.; Wise, D. R.; Thompson, C. B.; Kung, H. F. Synthesis of Optically Pure 4-Fluoro-Glutamines as Potential Metabolic Imaging Agents for Tumors. J. Am. Chem. Soc. 2011, 133, 1122-1133. 
14. Wood, J. M.; Furkert, D. P.; Brimble, M. A. Total Synthesis and Stereochemical Revision of the 2-Formylpyrrole Alkaloid Hemerocallisamine I. J. Nat. Prod. 2017, 80, 1926-1929.

15. Bahadoor, A.; Brauer, E. K.; Bosnich, W.; Schneiderman, D.; Johnston, A.; Aubin, Y.; Blackwell, B.; Melanson, J. E.; Harris, L. J. Gramillin A and B: Cyclic Lipopeptides Identified as the Nonribosomal Biosynthetic Products of Fusarium graminearum. J. Am. Chem. Soc. 2019, 140, 16783-16791.

16. Li, Y.; Lan, N.; Xu, L.; Yue, Q. Biosynthesis of Pneumocandin Lipopeptides and Perspectives for Its Production and Related Echinocandins. Appl. Microbiol. Biotechnol. 2018, 102, 9881-9891.

17. Yamaguchi, K.; Tsuji, T.; Wakuri, S.; Yazawa, K.; Kondo, K.; Shigemori, H.; Kobayashi, J. Stimulation of Nerve Growth Factor Synthesis and Secretion by Fellutamide A in Vitro. Biosci. Biotech. Biochem. 1993, 57, 195-199.

18. Cacho, R. A.; Jiang, W.; Chooi, Y.-H.; Walsh, C. T.; Tang, Y. Identification and Characterization of the Echinocandin B Biosynthetic Gene Cluster from Emericella rugulosa NRRL 11440. J. Am. Chem. Soc. 2012, 134, 16781-16790.

19. Franke, J.; Hertweck, C. Biomimetic Thioesters as Probes for Enzymatic Assembly Lines: Synthesis, Applications, and Challenges. Cell Chem. Biol. 2016, 23, 1179-1192. 